\title{
Challenge - o silêncio mata: mídias sociais e informação no combate à violência
}

\section{contra a mulher}

\author{
Challenge - silence kills: social media and information in the fight against violence against women \\ Challenge - el silencio mata: redes sociales e información en la lucha contra la violencia contra las
}

mujeres

Recebido: 09/07/2021 | Revisado: 17/07/2021 | Aceito: 21/07/2021 | Publicado: 28/07/2021

\author{
Ana Andreza Albuquerque Medeiros \\ ORCID: https://orcid.org/0000-0002-4012-883X \\ Instituto de Educação Superior do Vale do Parnaíba, Brasil \\ E-mail: andrezaaaam13@gmail.com \\ Anna Priscylla Pinheiro Diogenes Lima \\ ORCID: https://orcid.org/0000-0003-1121-6475 \\ Instituto de Educação Superior do Vale do Parnaíba, Brasil \\ E-mail: priscylladiogenes@gmail.com \\ Maria Eduarda Campos Bezerra \\ ORCID: https://orcid.org/0000-0003-2773-279X \\ Instituto de Educação Superior do Vale do Parnaíba, Brasil \\ E-mail: mariaeduardacbezerra@gmail.com \\ Vitória Lorrana Nunes da Silva \\ ORCID: https://orcid.org/0000-0002-5210-5520 \\ Instituto de Educação Superior do Vale do Parnaíba, Brasil \\ E-mail: vitoria.123.vlnds@gmail.com \\ Brenda Ellen Meneses Cardoso \\ ORCID: https://orcid.org/0000-0002-5982-5985 \\ Instituto de Educação Superior do Vale do Parnaíba, Brasil \\ E-mail: cbrendaellen@ hotmail.com \\ Ayane Araújo Rodrigues \\ ORCID: https://orcid.org/0000-0001-7792-6993 \\ Instituto de Educação Superior do Vale do Parnaíba, Brasil \\ E-mail: ayane.rodrigues@iesvap.edu.br \\ Thiago de Souza Lopes Araújo \\ ORCID: https://orcid.org/0000-0001-8543-8498 \\ Instituto de Educação Superior do Vale do Parnaíba, Brasil \\ E-mail: thiago.araujo@iesvap.edu.br
}

\begin{abstract}
Resumo
Objetivo: relatar a experiência acerca do uso de uma Tecnologia Digital da Informação e Comunicação como uma medida de prevenção e combate à violência contra a mulher, a qual se intensificou no período da pandemia da COVID-19, durante o isolamento social. Metodologia: esta produção científica de caráter qualitativo corresponde a um relato de experiência, cujo intuito é expor o processo de criação e implementação de uma tecnologia intervencionista, durante o período de fevereiro a junho do ano de 2020, resultado de um projeto de intervenção à violência praticada contra a mulher. Resultados: o "Challenge: o silêncio mata" expõe os principais canais de denúncia que estão disponíveis à vítima e a terceiros, e se mostrou eficaz no incentivo à queixa ao agressor por parte de quem sofre a violência, como também de pessoas que presenciam os casos violentos, possuindo repercussões significativas. Conclusão: A violência contra a mulher é um tema que, embora seja muito discutido, ainda deve ser explorado através de ferramentas que sejam capazes de impactar o público em geral. Assim, a proposta do trabalho foi expor dados alarmantes dos índices de agressão praticada contra a mulher e seu aumento no período de isolamento social, incentivando não só debates acerca do tema, mas também denúncia dos casos.
\end{abstract}

Palavras-chave: Cultura; Violência contra a mulher; Iniquidade de gênero; Isolamento social; Pandemias.

\begin{abstract}
Objective: to report the experience regarding the use of a Digital Information and Communication Technology as a measure to prevent and combat violence against women, which intensified during the period of the COVID-19 pandemic, during social isolation. Methodology: this qualitative scientific production corresponds to an experience report, whose purpose is to expose the process of creation and implementation of an interventionist technology, during the period from February to June of 2020, the result of an intervention project against violence practiced against women. Results: the "Challenge: silence kills" exposes the main reporting channels that are available to the victim and
\end{abstract}


third parties, and proved to be effective in encouraging the abuser to complain to the perpetrator, as well as people who witness the violent cases, having significant repercussions. Conclusion: Violence against women is a topic that, despite being much discussed, still needs to be explored through tools that are capable of impacting the general public. Thus, the proposal of the work was to expose alarming data on the rates of aggression practiced against women and its increase in the period of social isolation, encouraging not only debates on the subject, but also denunciation of cases.

Keywords: Culture; Violence against women; Gender inequity; Social isolation; Pandemics.

\section{Resumen}

Objetivo: reportar la experiencia sobre el uso de una Tecnología de Información y Comunicación Digital como medida para prevenir y combatir la violencia contra las mujeres, que se intensificó durante el período de la pandemia COVID-19, durante el aislamiento social. Metodología: esta producción científica cualitativa corresponde a un relato de experiencia, cuyo propósito es exponer el proceso de creación e implementación de una tecnología intervencionista, durante el período de febrero a junio de 2020, resultado de un proyecto de intervención contra la violencia practicada contra las mujeres. Resultados: el "Challenge: el silencio mata" expone los principales canales de denuncia que se encuentran a disposición de la víctima y terceros, y demostró ser efectivo para alentar al abusador a quejarse ante el perpetrador, así como ante las personas que presencian los casos violentos, habiendo repercusiones significativas. Conclusión: La violencia contra las mujeres es un tema que, a pesar de ser muy discutido, aún necesita ser explorado a través de herramientas que sean capaces de impactar al público en general. Así, la propuesta del trabajo fue exponer datos alarmantes sobre los índices de agresión practicada contra las mujeres y su incremento en el período de aislamiento social, incentivando no solo debates sobre el tema, sino también denuncia de casos.

Palabras clave: Cultura; La violencia contra las mujeres; Desigualdad de género; Aislamiento social; Pandemias.

\section{Introdução}

A violência contra a mulher é um problema de saúde pública com impacto global, que apresenta grandes repercussões não só para as vítimas, mas também para as famílias e comunidades. Segundo a Organização Mundial da Saúde (OMS) esse tipo de violência pode se manifestar de várias maneiras, dentre elas destacam-se a violência física (agressões, assassinato, tortura), a violência psicológica (proferir palavrões, ameaçar, intimidar, manipular, humilhar), violência patrimonial (obsessão em controlar as finanças, confisco de documentos e dinheiro), violência sexual (assédio, relações sexuais sem consentimento) e stalking (obsessão em controlar redes sociais, mensagens de texto ou telefonemas; perseguir) (Viero et al., 2021).

Em particular, a violência doméstica é a que possui maior prevalência na esfera social, sendo baseada em um poder e controle exercido pelo próprio parceiro da vítima (Gulati \& Kelly, 2020). Estudos apontam que mais de um terço dos casos de feminicídio são causados pelo companheiro da mulher e que uma a cada três mulheres que estão em idade reprodutiva já sofreu, em algum momento da vida, violência praticada por seu parceiro, seja ela sexual ou física (Stöckl et al., 2013).

Diante disso, com o isolamento social imposto no mundo todo, em decorrência da pandemia da COVID-19 (doença causada pelo coronavírus, denominado SARS-CoV-2), a violência doméstica passou a ser uma preocupação de várias organizações, bem como de representantes da sociedade civil e de pesquisadores, tendo em vista que em vários países houve um aumento significativo dos índices de violência contra as mulheres em seu próprio domicílio (Sánchez et al., 2020). Desde o início da pandemia, países como a Itália, Espanha, França, Estados Unidos e China, vivenciaram um crescimento considerável de registros policiais de violência doméstica. Enquanto isso, no Brasil, conforme a Ouvidoria Nacional dos Direitos Humanos (ONDH), canal de denúncia do Ministério da Mulher, da Família e dos Direitos Humanos (MMFDH), no mês de março, mês dedicado nacionalmente à mulher, entre os dias $1^{\circ}$ e $25^{\circ}$ houve um acréscimo de $18 \%$ dos índices de denúncias que foram registradas pelo Ligue 180 e Disque 100 (Vieira et al., 2020).

As Tecnologias Digitais de Informação e Comunicação (TDICs) correspondem a sistemas de transmissão e telecomunicações, software, serviços de telecomunicação (sem fio, móvel, fixo), hardware de computador, produção de conteúdo e gerenciamento de sistemas multimídia, redes e serviços, aplicativos de dispositivo móvel e tecnologias da Internet. Neste certame, esses instrumentos têm apresentado significativo impacto na área da saúde, conforme a publicação do relatório "cruzando o quiasma da qualidade", a partir do Instituto de Medicina (IOM), no ano de 2001, demonstrando um maior desenvolvimento e disseminação do emprego das tecnologias da informação e comunicação, melhorando as condições de 
saúde e segurança do paciente. Isso tem sido feito através da redução de falhas humanas, aperfeiçoamento de resultados clínicos, coordenação eficaz do cuidado e armazenamento seguro dos dados no decorrer dos anos (Alotaibi \& Federico, 2017; Alturkistani et al., 2018; Joseph-Shehu et al., 2019; Posadzki et al., 2016).

Nesse âmbito, a vídeogravação é uma TDIC de grande destaque nos dias atuais, mesmo não sendo uma tecnologia recente. Ela oferece mínima dificuldade no que tange à operacionalização, não necessitando de um conhecimento abrangente para entender o objetivo de aprendizagem que foi extraído, além de ser um recurso prático, seguro e de baixo custo (Donkin et al., 2019; Sugamoto et al., 2015).

Assim, o objetivo deste trabalho é relatar a experiência acerca do uso de uma Tecnologia Digital da Informação e Comunicação, uma gravação de vídeo no formato de challenge, modalidade baseada na produção de vídeos curtos, nos quais os participantes gesticulam ou fazem uso de artefatos (maquiagens, acessórios, roupas, entre outros), com o fito de causar uma reflexão a respeito de determinada temática (Borges et al., 2020; Cardoso, 2019). Nesse sentido, sendo usado como uma medida de prevenção e combate à violência contra a mulher, a qual se intensificou no período da pandemia da COVID-19, durante o isolamento social.

\section{Metodologia}

Esta produção científica de caráter qualitativo corresponde a um relato de experiência, cujo intuito foi expor o processo de criação e implementação de uma tecnologia intervencionista, durante o período de fevereiro a junho do ano de 2020, resultado de um projeto de intervenção à violência praticada contra a mulher, referente às disciplinas Integração-EnsinoServiço e Comunidade II e Métodos de Estudo e Pesquisa II, do curso de medicina da Faculdade de Ciências Humanas, Exatas e da Saúde do Piauí/Instituto de Educação Superior do Vale do Parnaíba (FAHESP/IESVAP). A pesquisa qualitativa, através de dados descritivos obtidos por meio da observação, permite a apresentação de uma prática, bem como proporciona a reflexão acerca dessa prática, com ênfase social no objetivo de estudo (Pereira et al., 2018).

Utilizando uma abordagem do problema baseada no arco de Maguerez, a produção deste trabalho científico foi dividida em cinco etapas: (1) observação da realidade, (2) pontos-chave, (3) teorização, (4) hipóteses de solução e (5) aplicação à realidade (Carabetta, 2016).

A primeira etapa foi a escolha do tema explorado ao longo do projeto, delimitado a partir da análise dos dados fornecidos pelo Fórum Brasileiro de Segurança Pública, que demonstram o aumento significativo dos casos de feminicídio em 12 estados brasileiros nos meses de março e abril de 2020, devido à pandemia da COVID-19 (Brasil, 2020). Já a segunda etapa consistiu em determinar os principais determinantes que englobam o problema a seguir, são eles: o patriarcalismo, a cultura machista, comunicação limitada da vítima para com terceiros, ameaças por parte do agressor e sentimentos de vergonha e medo vivenciados pela mulher.

Em seguida, no terceiro passo, foi obtido o embasamento teórico por meio das bases de dados Scielo, PubMed e LILACS, de onde foram selecionadas publicações a partir do ano de 2016 até o ano de 2021, com exceção de um artigo publicado no ano de 2013 e de outro em 2015. Dessa forma, através de uma busca ampla, foram encontrados 27 artigos, com exclusão de cinco produções sob os critérios de idioma, ano de publicação e não correspondência à temática, resultando em um referencial teórico elaborado a partir de 23 artigos científicos. Já os Descritores em Ciências da Saúde (DeCS) utilizados no trabalho foram "cultura", "violência contra a mulher", "iniquidade de gênero", "isolamento social", e "pandemias". A busca na literatura direcionou as principais temáticas expressas no presente trabalho, como: o aumento dos índices de violência contra a mulher e sua relação com o isolamento social causado pela pandemia do novo coronavírus, a dificuldade de denúncia devido à impunidade, receio de contaminação pelo vírus da COVID-19 e temor do agressor, além da influência do patriarcalismo, o qual inferioriza e subordina as mulheres aos homens, aumentando a posse e o controle sobre a mulher. 
Posteriormente, na quarta etapa, iniciou-se o planejamento do "Challenge: o silêncio mata". Nessa fase, ocorreu a definição da função de cada integrante na construção do trabalho, como editor, narrador e figurinista (Aragão et al., 2019). Em seguida, baseado em dados estatísticos e outras informações acerca da violência contra a mulher, criou-se o roteiro (Figura 1), uma estruturação escrita de cenas da história narrada, a qual usa descrições de sons e imagens detalhadas, sendo composta por sequência, programação, imagem, aspectos cenográficos, elementos audiovisuais, sugestões relevantes e tempo de duração, em segundos, da sequência (Oechler et al., 2017).

Figura 1. Representação gráfica do roteiro seguido na construção do Challenge.

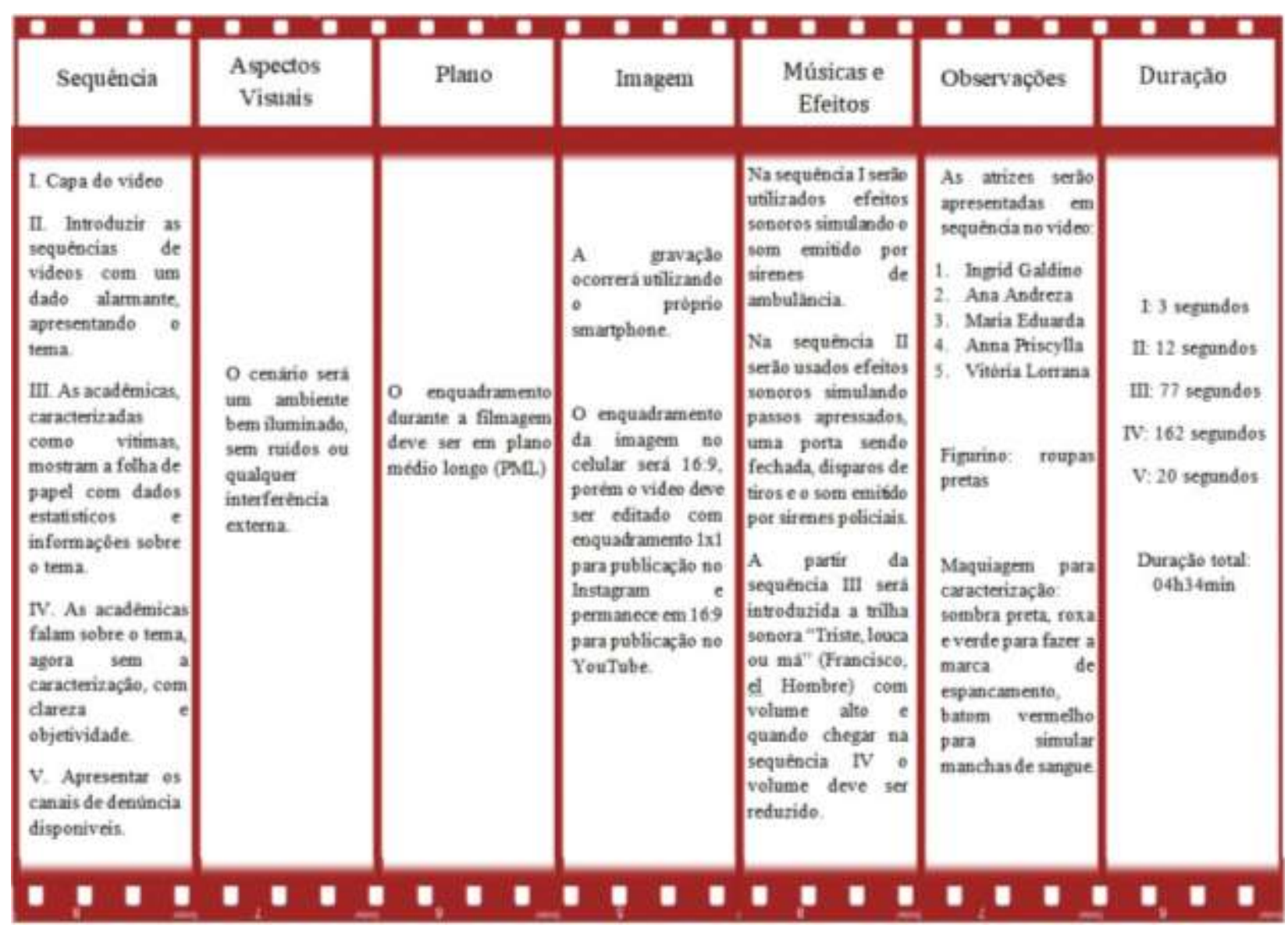

Fonte: Autores.

Subsequentemente, sucedeu-se a edição do vídeo utilizando o aplicativo InShot, a partir da junção dos cinco vídeos produzidos individualmente por cada acadêmica. Cada integrante enviou seu vídeo à acadêmica responsável pela edição do challenge, que, por sua vez, associou os vídeos e introduziu a trilha sonora "Triste, Louca ou Má", canção de Francisco, el Hombre, aplicando efeitos sonoros de impacto, simulando sirenes, gritos, choros e tiros.

E, por último, durante a quinta etapa do arco, ocorreu a exposição da tecnologia desenvolvida e utilizada como projeto de intervenção em plataformas de grande utilização nos dias atuais, como Instagram e YouTube. Nas publicações, foram observados dados como alcance do público-alvo, quantidade de curtidas, comentários, número total de visualizações e compartilhamentos entre os usuários.

\section{Resultados e Discussão}

O isolamento social causado pela pandemia do novo coronavírus levou ao aumento do convívio, expandindo as chances de causar estresse nas relações interpessoais e estimular os desgastes familiares, inclusive da mulher com o agressor. 
Instabilidades econômicas foram exacerbadas nesse período, como o desemprego; situação que fortaleceu a dependência econômica das mulheres e intimidou a figura masculina construída culturalmente como provedor do lar. Tal realidade amplifica a probabilidade do emprego da violência intrafamiliar como meio de validação do poder masculino. Além desses, vários outros fatores contribuíram para complicar os vínculos familiares; circunstância que traz resultados intensamente desfavoráveis para as mulheres (Emezue, 2020; Ferreira et al., 2020).

À medida que houve o aumento do número de indivíduos em domicílio e do tempo de estadia em casa, sucedeu-se também o excesso de trabalho das mulheres por conta do acúmulo dos afazeres domésticos e de cuidados com os filhos. Muitas vezes, se alia a isso, ainda, a nova realidade de trazer o trabalho para o lar (home office), o que o torna um local de produção e reprodução social, sem condições apropriadas para essa aplicabilidade (Marques et al., 2020).

A restrição ao espaço privado restringiu a relação das mulheres com sua rede coletiva de apoio, como parentes, colegas de trabalho, comunidade em que vive e equipamentos sociais no território, o que diminuiu as possibilidades de reconhecimento e confronto do problema da violência. Devido ao isolamento social, o acesso aos serviços de apoio às mulheres em situação de violência e vulnerabilidade também ficou afetado. Isso colaborou não só para a persistência e acentuação das situações de violência, mas também para as transgressões que passaram a ocorrer na pandemia, o que demonstra a carência na adoção e reforço de ferramentas no enfrentamento do problema (Roesch et al., 2020).

Assim, como forma de tentar reverter a problemática supracitada e evitar suas consequências, foi desenvolvida uma TDIC, um elemento básico da vida contemporânea que se refere à convergência de setores de tecnologia da informação, telecomunicações e tecnologia de mídia baseada em tecnologia digital (Magalhães et al., 2020). Elas estão em uso desde a segunda metade dos anos noventa e nos dias atuais é uma realidade que permite aos usuários produzir, armazenar, transmitir e manejar informações, através do processamento e transmissão de áudio e vídeo, meios de difusão, redes de controle e monitorização (Dogba et al., 2019).

$\mathrm{Na}$ área da saúde, suas aplicações se estendem a comunicação em tempo real com indivíduos nas comunidades virtuais, ao acesso de registros médicos, busca de informações sobre saúde e serviços de saúde, além de ser útil na transmissão de dados ou envio de mensagens pela web ou telefone. Além disso, estudos têm identificado a potencialidade dessas tecnologias em serviços de prevenção e promoção da saúde (Posadzki et al., 2016).

Isso foi mostrado na nossa experiência durante a produção de uma Tecnologia Digital da Informação e Comunicação como medida de combate à violência contra à mulher e prevenção de novos casos. Dentre as potencialidades da nossa TDIC produzida, destacam-se o apoio social e o encorajamento à denúncia, destinados às mulheres que diariamente são vítimas de violência domiciliar e que não contam com a ajuda de pessoas próximas por medo de julgamentos ou de serem descobertas por seu agressor. Além disso, essa ferramenta também traz informações importantes sobre os tipos de violência e como elas podem ser identificadas, já que muitas vezes a identificação é dificultada devido à visão de ser algo intrínseco ao homem, caracterizando sua personalidade como agressiva e imutável. Outro aspecto oferecido por essa tecnologia é apresentar os principais canais de denúncia disponíveis, facilitando a queixa ao agressor por parte não só das vítimas, como também de testemunhas de casos de violência.

Nesse sentido, após a publicação do vídeo no Instagram e YouTube, evidenciou-se um grande engajamento do público usuário dessas plataformas, através dos números elevados de visualizações (836), alcance (1079), curtidas (299), comentários (87) e compartilhamentos (12) (Gráfico 1). 
Gráfico 1. O alcance do total dos conteúdos produzidos e publicados nas redes sociais (Instagram e YouTube) ao longo dos quatro meses.

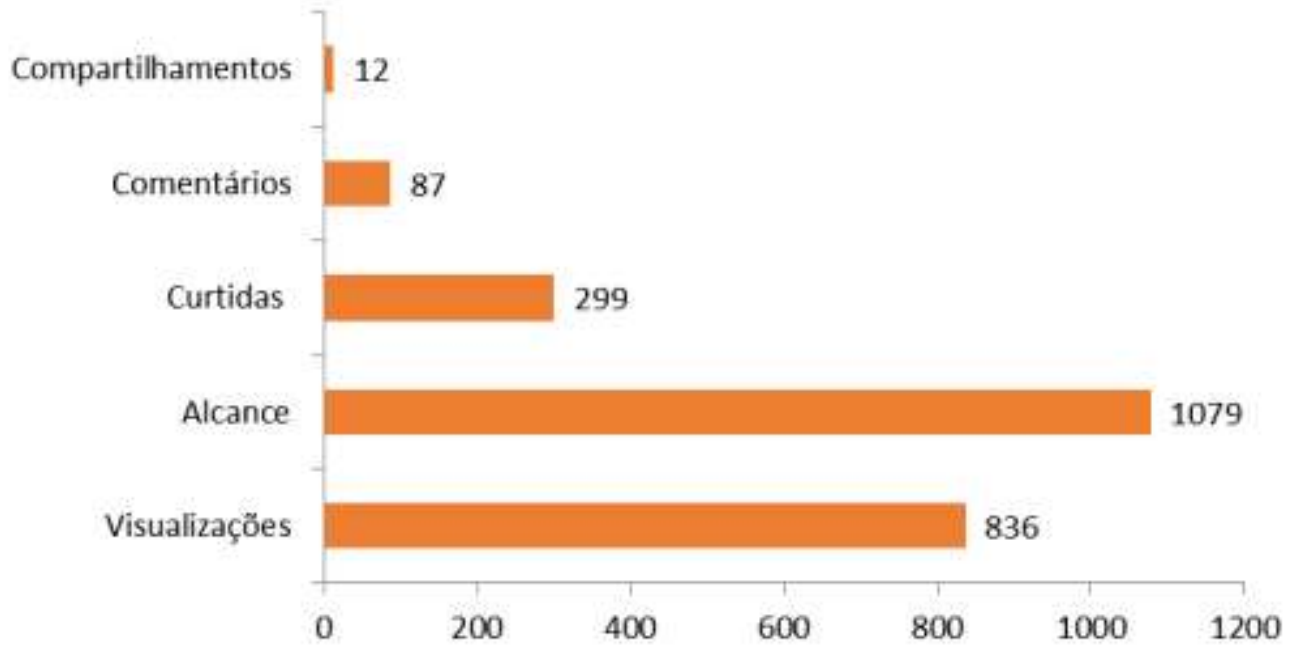

Fonte: Autores.

Dessa forma, nosso projeto de intervenção utilizando uma vídeogravação publicada em redes sociais de grande alcance social cumpriu com seu objetivo pré-estabelecido de incitar o engajamento do público e fazê-lo notar a importância de discutir acerca da violência contra a mulher, incentivando denúncias desse crime, além de informar sobre o aumento de casos durante o isolamento social causado pela COVID-19. Logo, foi possível difundir a temática e demonstrar sua relevância, tendo grandes repercussões positivas para a sociedade.

\section{Conclusão}

Conclui-se, portanto, que a violência contra a mulher é um tema que, embora seja muito discutido na sociedade, ainda deve ser explorado através de ferramentas que sejam capazes de impactar o público em geral. Assim, a proposta do trabalho foi expor dados alarmantes dos índices da violência praticada contra a mulher e seu aumento no período de isolamento social, incentivando não só debates acerca do tema, mas também denúncia dos casos. Somando-se a isso, o projeto tem a importância de estimular a autorreflexão das acadêmicas, para que se tornem profissionais humanizadas, empáticas e com senso crítico apurado. Além disso, a TDIC foi um ponto chave para o desenvolvimento do projeto, uma vez que a internet foi o maior aparato utilizado por todos no momento de isolamento social, sendo de fácil acesso e de grande poder de disseminação, o qual ajudou no entendimento sobre o impacto de uma agressão, seja ela psicológica, física, mental e/ou sexual, assim como tornou mais evidente as formas de denúncia, encorajando a vítima e as pessoas da sua convivência a delatar o agressor.

Logo, sugere-se, com este trabalho, um maior número de produções acadêmicas a respeito do aumento da violência contra a mulher, observado durante a pandemia da COVID-19, descrevendo outros tipos de TDICs que podem ser utilizadas como forma de combate a esse tipo de violência. Além disso, também é possível explorar a utilização de TDICs em projetos sociais de eixos diversificados (violência infantil, do idoso, de pessoas com problemas mentais, violência contra os animais), atribuindo essa tecnologia como um suporte de conscientização e adesão, bem como instrumento de denúncia de atos criminosos referentes à causa em defesa.

\section{Referências}

Alotaibi, Y. K., \& Federico, F. (2017). The impact of health information technology on patient safety. Saudi Medical Journal, 38(12), 1173-1180. 
Alturkistani, A., Majeed, A., Car, J., Brindley, D., Wells, G., \& Meinert, E. (2018). Health information technology uses for primary prevention in preventive medicine: a scoping review protocol. BMJ Open, 8(9), e023428.

Aragão, J.C.S., Soares G.A.R., Santos I.X.P., Souza M.C., \& Souza, R.S. (2019). Produção De Vídeos Como Material Didático De Apoio Para Aprendizagem Em Saúde Da Mulher: Relato De Experiência. Revista Práxis, 11(22).

Borges, I. S. C., Vieira, A. C. N., Oliveira, R. M., Silva, G. M. e, \& Raimondi, G. A. (2020). Representatividade LGBT+ na Educação Médica e Covid-19: Construindo Redes de Cuidado e Solidariedade. Revista Brasileira de Educação Médica, 44(suppl 1).

Brasil. (2020). Violência doméstica durante a pandemia de Covid-19. Fórum Brasileiro de Segurança Pública. https://forumseguranca.org.br/wpcontent/uploads/2018/05/violencia-domestica-covid-19-v3.pdf

Carabetta, V., Jr. (2016). Metodologia ativa na educação médica. Revista de Medicina, 95(3), 113.

Cardoso, B. (2019). O que é Evoluiu Challenge? Entenda o vídeo que virou moda no Instagram. TechTudo. https://www.techtudo.com.br/noticias/2019/09/oque-e-evoluiu-challenge-entenda-o-video-que-virou-moda-no-instagram.ghtml

Dogba, M. J., Dossa, A. R., Breton, E., \& Gandonou-Migan, R. (2019). Using information and communication technologies to involve patients and the public in health education in rural and remote areas: a scoping review. BMC Health Services Research, 19(1).

Donkin, R., Askew, E., \& Stevenson, H. (2019). Video feedback and e-Learning enhances laboratory skills and engagement in medical laboratory science students. BMC Medical Education, 19(1).

Emezue, C. (2020). Digital or Digitally Delivered Responses to Domestic and Intimate Partner Violence During COVID-19. JMIR Public Health and Surveillance, 6(3), e19831.

Ferreira, V. C., Silva, M. R. F. da, Montovani, E. H., Colares, L. G., Ribeiro, A. A., \& Stofel, N. S. (2020). Saúde da Mulher, Gênero, Políticas Públicas e Educação Médica: Agravos no Contexto de Pandemia. Revista Brasileira de Educação Médica, 44(suppl 1).

Gulati, G., \& Kelly, B. D. (2020). Domestic violence against women and the COVID-19 pandemic: What is the role of psychiatry? International Journal of Law and Psychiatry, 71, 101594.

Joseph-Shehu, E. M., Ncama, B. P., Mooi, N., \& Mashamba-Thompson, T. P. (2019). The use of information and communication technologies to promote healthy lifestyle behaviour: a systematic scoping review. BMJ Open, 9(10), e029872.

Magalhães, A. J. de A., Rocha, M. H. A., Santos, S. C., Dantas, C. B., Manso, G. J. de M. C., \& Ferreira, M. D. A. (2020). O Ensino da Anamnese Assistido por Tecnologias Digitais durante a Pandemia da Covid-19 no Brasil. Revista Brasileira de Educação Médica, 44(suppl 1).

Marques, E. S., Moraes, C. L. de, Hasselmann, M. H., Deslandes, S. F., \& Reichenheim, M. E. (2020). A violência contra mulheres, crianças e adolescentes em tempos de pandemia pela COVID-19: panorama, motivações e formas de enfrentamento. Cadernos de Saúde Pública, 36(4).

Oechsler V., Fontes B.C., Borba M.C. (2017). Etapas da produção de vídeos por alunos da educação básica: uma experiência na aula de matemática. Revista Brasileira de Educação Básica. https://rbeducacaobasica.com.br/etapas-da-producao-de-videos-por-alunos-da-educacao-basica-uma-experiencia-na-aula-dematematica/

Pereira, A. S., Shitsuka, D. M., Parreira, F. J., \& Shitsuka, R. (2018). Metodologia da pesquisa científica. [free e-book]. Santa Maria/RS. Ed. UAB/NTE/UFSM.

Posadzki, P., Mastellos, N., Ryan, R., Gunn, L. H., Felix, L. M., Pappas, Y., Gagnon, M.-P., Julious, S. A., Xiang, L., Oldenburg, B., \& Car, J. (2016). Automated telephone communication systems for preventive healthcare and management of long-term conditions. Cochrane Database of Systematic Reviews, 2016(12).

Roesch, E., Amin, A., Gupta, J., \& García-Moreno, C. (2020). Violence against women during covid-19 pandemic restrictions. BMJ, m1712.

Sánchez, O. R., Vale, D. B., Rodrigues, L., \& Surita, F. G. (2020). Violence against women during the COVID-19 pandemic: An integrative review. International Journal of Gynecology \& Obstetrics, 151(2), 180-187.

Stöckl, H., Devries, K., Rotstein, A., Abrahams, N., Campbell, J., Watts, C., \& Moreno, C. G. (2013). The global prevalence of intimate partner homicide: a systematic review. The Lancet, 382(9895), 859-865.

Sugamoto, Y., Hamamoto, Y., Kimura, M., Fukunaga, T., Tasaki, K., Asai, Y., Takeshita, N., Maruyama, T., Hosokawa, T., Tamachi, T., Aoyama, H., \& Matsubara, H. (2015). A Novel Method for Real-Time Audio Recording With Intraoperative Video. Journal of Surgical Education, 72(5), 795-802.

Vieira, P. R., Garcia, L. P., \& Maciel, E. L. N. (2020). Isolamento social e o aumento da violência doméstica: o que isso nos revela? Revista Brasileira de Epidemiologia, 23.

Viero, A., Barbara, G., Montisci, M., Kustermann, K., \& Cattaneo, C. (2021). Violence against women in the Covid-19 pandemic: A review of the literature and a call for shared strategies to tackle health and social emergencies. Forensic Science International, 319, 110650. 